\title{
The efficacy and safety of immunotherapy in thymic epithelial tumors: more effective, more risky: a systematic review
}

\author{
Xinyu Song ${ }^{1,2}$, Jiang Fan $^{3}$, Liang Zhu ${ }^{1,2}$, Zhehai Wang ${ }^{4}$, Yayi He ${ }^{1,2}$, Caicun Zhou ${ }^{1,2}$ \\ ${ }^{1}$ Department of Medical Oncology, Shanghai Pulmonary Hospital, School of Medicine, Tongji University, Shanghai, China; ${ }^{2}$ Tongji University, \\ Shanghai, China; ${ }^{3}$ Department of Thoracic Surgery, Shanghai First People's Hospital, First People's Hospital Affiliated to Shanghai Jiao Tong \\ University, Shanghai, China; ${ }^{4}$ Department of Medical Oncology, Shandong Cancer Hospital, Jinan, China \\ Contributions: (I) Conception and design: X Song, Y He, Z Wang; (II) Administrative support: C Zhou; (III) Provision of study materials or patients: \\ X Song, J Fan; (IV) Collection and assembly of data: X Song, L Zhu; (V) Data analysis and interpretation: X Song; (VI) Manuscript writing: All \\ authors; (VII) Final approval of manuscript: All authors. \\ Correspondence to: Zhehai Wang. Department of Medical Oncology, Shandong Cancer Hospital, No. 440 Jiyan Road, Jinan, Shandong Province \\ 250117, China. Email: wzhai8778@sina.com; Yayi He; Caicun Zhou. Department of Medical Oncology, Shanghai Pulmonary Hospital, School of \\ Medicine, Tongji University, No. 507 Zhengmin Road, Shanghai 200433, China. Email 2250601@qq.com; caicunzhoudr@126.com.
}

Background: Thymic epithelial tumors (TETs) are rare malignant neoplasms originating from thymic epithelial cells. The current treatment for localized TETs is surgical removal. However, 20-30\% of thymomas and $70-80 \%$ of thymic carcinomas are unresectable, recurrent, or metastatic at the time of detection. The standard therapy for these patients is chemotherapy, but the effect is limited. With a deeper understanding of tumor immunity, immunotherapy for various cancers has rapidly developed. Antibodies against cytotoxic T-lymphocyte antigen-4, programmed death-1, and programmed death-ligand 1 have been approved for the treatment of many solid tumors. Compared with traditional treatments, these immune checkpoint inhibitors (ICIs) have better efficacy and lower toxicity. Recently, ICIs have been used more enthusiastically in the treatment of TETs. However, due to the unique biological characteristics of the thymus, immunotherapy usually causes severe immune-related adverse events (irAEs). Most previous studies on immunotherapy in TETs had small sample sizes and reported diverse conclusions.

Methods: We collected relevant studies in PubMed during the last five years and analyzed the available data to discuss the efficacy and safety of ICIs in TETs.

Results: According to 14 previous studies in the past five years, all TETs showed expression of programmed death-ligand 1, while thymic carcinomas showed $100 \%$ expression. The best median progression-free survival (mPFS) among the five studies was 6.5 months, and the best median overall survival (mOS) was 24.9 months. In addition, the most common irAEs were myasthenic symptoms, liver enzyme elevation, and elevated creatine phosphokinase levels.

Conclusions: ICIs can be used in TET treatment, especially for thymic carcinomas, in the absence of standard second-line treatment. However, more attention should be paid to irAEs.

Keywords: Thymic epithelial tumors (TETs); PD-L1; immune checkpoint inhibitors (ICIs); efficacy; safety

Submitted Feb 16, 2021. Accepted for publication Jun 25, 2021.

doi: $10.21037 /$ jtd-21-290

View this article at: https://dx.doi.org/10.21037/jtd-21-290

(c) Journal of Thoracic Disease. All rights reserved. 


\section{Introduction}

Thymic epithelial tumors (TETs) are rare thoracic carcinomas (1), and the annual incidence is reported to be between $1.3 \%$ and $3.2 \%$ (2). Most patients present with chest pain or chest discomfort; some patients may have emaciation, night sweats, cough, dyspnea, and other symptoms. If the tumor is large, a superior vena cava obstruction may occur. TETs are divided into two broad categories: thymoma (TM) and thymic carcinoma (TC). TM is a common primary neoplasm of the anterior mediastinum. TC is an invasive tumor that often metastasizes to regional lymph nodes and extrathoracic organs. Therefore, the prognosis of TC is worse than that of TM. The 5 -year survival rate is approximately $90 \%$ for TMs, while it is only $55 \%$ for TCs (2). The World Health Organization (WHO) histological classification of thymoma is generally considered to have a guiding significance for predicting TM recurrence. According to the WHO histological classification, Wright et al. classified TMs into five types, $\mathrm{A}, \mathrm{AB}, \mathrm{B} 1, \mathrm{~B} 2, \mathrm{~B} 3$, and $\mathrm{C}$, respectively, depending on their increasing local aggressiveness and recurrence rate (3). However, type $\mathrm{C}$ was completely distinct from other types of TM, with significantly higher recurrence and metastasis rates ( $41 \%$ vs. 9.7\%) (4), and thus became an independent type called TC. The pathological classification was not only beneficial to the early diagnosis of TET but also to predict the malignant degree of TET to a certain extent, which was more beneficial to the later treatment of patients.

At present, complete surgical resection of the tumor is the most effective treatment option for localized TETs. Chemotherapy with or without radiotherapy is the only guideline-recommended treatment option for patients who have already metastasized tumors (5). Platinum-based chemotherapy is considered to be the most effective firstline chemotherapy (6). The objective response rate (ORR) of first-line platinum-based chemotherapy was $50 \%$ for TM and $20 \%$ for TC $(7,8)$. The ORR of second-line singledrug chemotherapy was less than $10 \%$ for TETs $(9,10)$. The ORR of sunitinib targeted therapy for TM and TC was $6 \%$ and $26 \%$, respectively (11). The ORR of everolimus targeted therapy was only $9 \%$ and $16 \%$, respectively (12). It can be seen that the effect of chemotherapy and targeted therapy in patients with TM/TC is very limited. To date, many clinical studies have shown that immunotherapy for TETs is currently under development. However, a considerable proportion of TET cases are complicated with autoimmune syndromes, such as myasthenia gravis and Hashimoto's thyroiditis. Although immunotherapy is promising, more attention should be paid to the occurrence of immune-related adverse events (irAEs) to balance the risk and efficacy of immunotherapy.

We know that the PD-1 and PD-L1 pathways are important for negatively regulating the immune escape from $T$ cells. The expression of PD-1/PD-L1 can be used as a prognostic marker in many malignant tumors (13-16). Many previous studies have shown that high expression of PD-L1 exists in various tumor cells, such as advanced NSCLC, melanoma, colorectal cancer, and glioma (17). The mechanism of PD-1-mediated immunosuppression is cross-correlated with multiple cellular signaling pathways. In general, the function of PD-L1 is to combine with PD-1, thus inhibiting $\mathrm{T}$ cell activation, to avoid autoimmune diseases caused by the over-activation of $\mathrm{T}$ cells. In the tumor microenvironment, $\mathrm{T}$ cells are responsible for inducing infiltration to have a high expression of PD-1, while the tumor cells express high levels of PD-L1, maintaining the PD-1/PD-L1 pathway in a state of continuous activation. In this case, the immunoreceptor tyrosine-based switch motif recruits phosphatases, such as Src homology 2 domain tyrosine phosphatases, and activates the renin-angiotensin system, phosphatidylinositol 3 kinase/ protein kinase B (PI3K/AKT), and other pathways. This results in the inhibition of downstream $\mathrm{T}$ cell proliferation and the generation of related cytokines, leading to the inhibition of $\mathrm{T}$ cell function and the transduction of negative signals, thus producing negative regulatory effects (18). In addition, T cell function can be inhibited by increasing the expression of the basic leucine zipper transcription factor ATF-like (19) (Figure 1).

Cytotoxic T-lymphocyte antigen-4 (CTLA-4, CD152) is an immune checkpoint molecule homologous to CD28, which can bind to B7-1 (CD80) and B7-2 (CD86) ligands. CTLA-4 is expressed at low levels on the surface of naive $\mathrm{T}$ cells, effector T cells, and regulatory $\mathrm{T}$ cells (20). CTLA4 transmits signals to downstream protein phosphatase $2 \mathrm{~A}, \mathrm{PI} 3 \mathrm{~K}$, and other molecules by binding CD 80 and CD86 $(21,22)$. CD28 is expressed on the surface of both resting and activated T cells, but CTLA-4 is expressed only on activated $\mathrm{T}$ cells (23). After $\mathrm{T}$ cells are activated, the mRNA of CTLA-4 is rapidly transcribed, the protein expression level begins to increase, and the expression level is then downregulated through endocytosis (24). CTLA-4 competitively binds to B7 ligands instead of CD28 and then becomes a stable polymeric complex with $\mathrm{B} 7$ in the form 


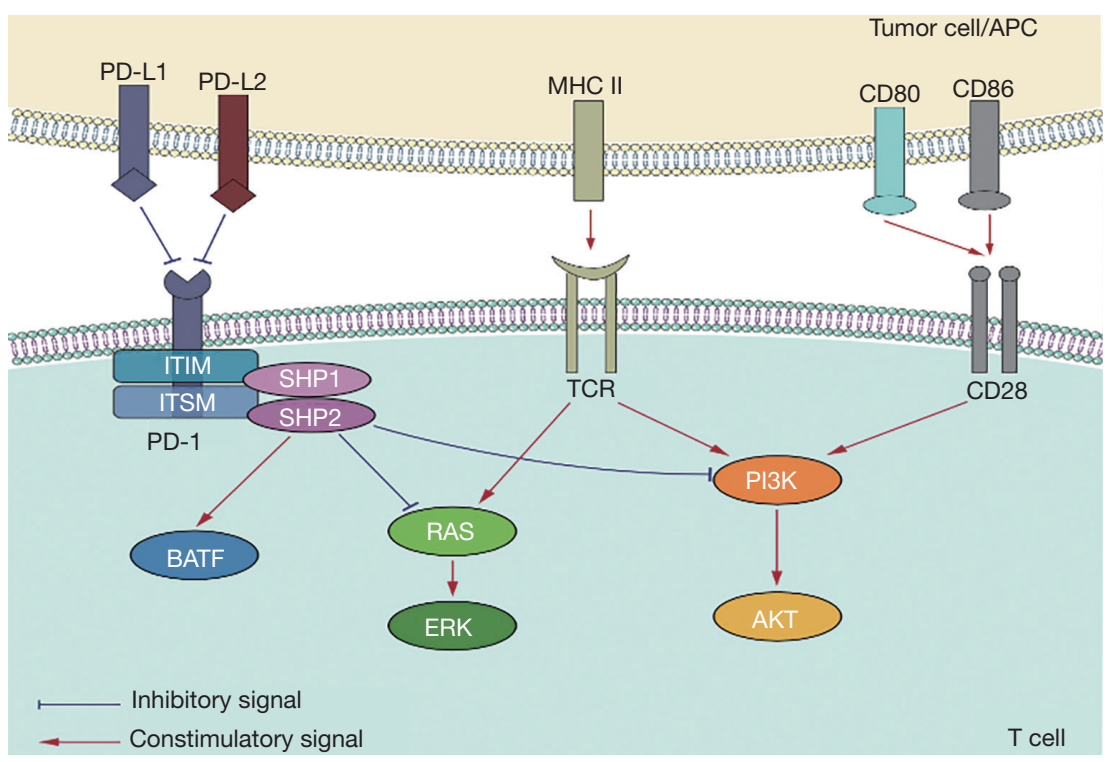

Figure 1 After PD-L1 binds to PD-1, phosphatases such as SHP2 are recruited in the structural domain and removed phosphokinases, thus inhibiting the transmission of positive signals from T cell receptors (TCR) and CD28, thereby affecting downstream PI3K-AKT, RAS and other pathways to inhibit $\mathrm{T}$ cell activation. In addition, the expression of BATF was also increased to inhibit $\mathrm{T}$ cell function.

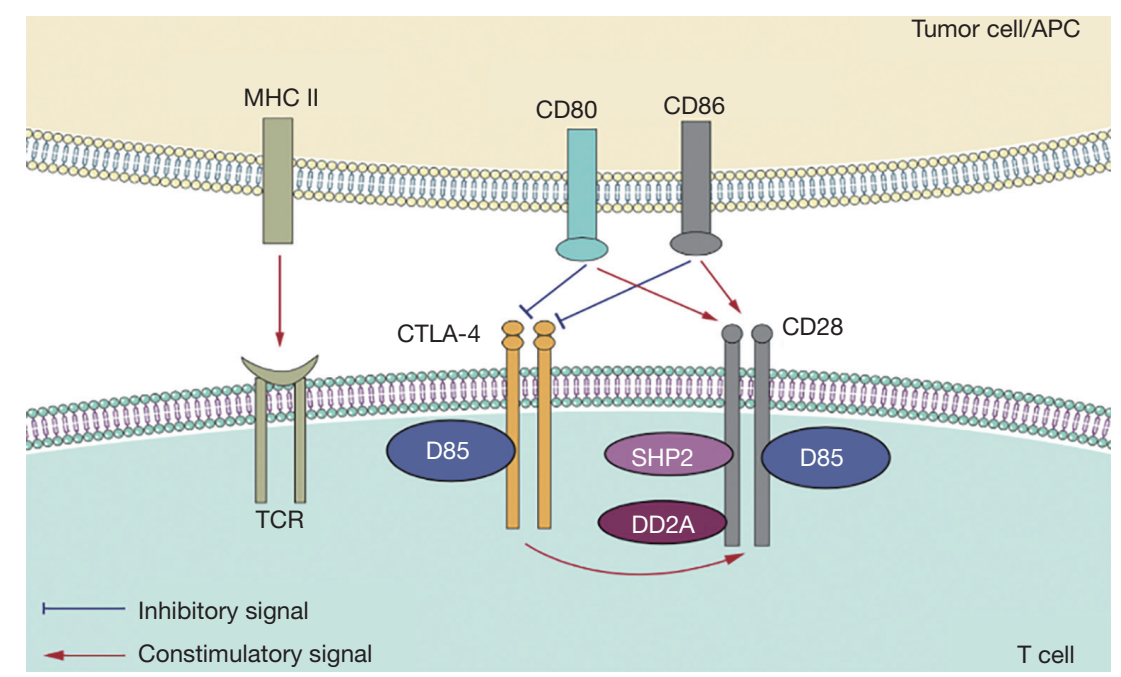

Figure 2 After CTLA-4 binds to B7-1 and B7-2, it transmitted signals to downstream protein PP2A, PI3K and other molecules. CTLA4 competitively combined B7 ligands with CD28, then blocked the CD28:B7 pathway. CTLA-4 also interfered with the formation of lipid rafts on the plasma membrane.

of a homologous dimer, blocking the CD28:B7 pathway, thus acting as a negative feedback regulatory molecule for $\mathrm{T}$ cell activation (25). CTLA-4 also interferes with the formation of lipid rafts on the plasma membrane and destroys the microenvironment required for CD28 (26). In addition, CTLA-4 increases the movement of T cells, thus reducing the contact time between $\mathrm{T}$ cells and antigenpresenting cells (APC), and increases the $\mathrm{T}$ cell activation "threshold" (27) (Figure 2).

We present the following article in accordance with the PRISMA reporting checklist (available at https://dx.doi. org/10.21037/jtd-21-290). 
Table 1 Profiles of PD-L1 expression in TETs

\begin{tabular}{|c|c|c|c|c|}
\hline Study & Years & Total cases & TMs/TCs & PD-L1 expression \\
\hline Judit Bedekovics, et al. (30) & 2020 & 36 & $29 / 7$ & $100 \%$ \\
\hline \multirow[t]{2}{*}{ Shunta Ishihara, et al. (31) } & 2020 & 66 & $53 / 11$ & TPS-V (\%) A:2; AB:2; B1:5; B2:16; B3:6; TC:11 \\
\hline & & & & TPS-IA (\%) A:18; AB:19; B1:25; B2:55; B3:78; TC:44 \\
\hline Rumi Higuchi, et al. (33) & 2019 & 39 & $31 / 8$ & $53.9 \%$ \\
\hline Joon Seon Song, et al. (34) & 2019 & 368 & $308 / 60$ & $90.6 \%$ \\
\hline Soichiro Funaki, et al. (35) & 2019 & 43 & $0 / 43$ & $60.5 \%$ \\
\hline Yanmei Chen, et al. (36) & 2018 & 70 & $50 / 20$ & A:36\%; AB:15\%; B1:33\%; B2:86\%; B3:90\%; TC:70\% \\
\hline Kathryn C. Arbour, et al. (39) & 2017 & 44 & $12 / 11$ & $65 \%$ \\
\hline Annikka Weissferdt, et al. (40) & 2017 & 100 & $74 / 26$ & $64 \% / 54 \%$ \\
\hline Alberto M. Marchevsky, et al. (41) & 2017 & 46 & $38 / 8$ & $92 \% / 50 \%$ \\
\hline Yuki Katsuya, et al. (42) & 2016 & 30 & $12 / 18$ & $67 \% / 41 \%$ \\
\hline
\end{tabular}

TET, thymic epithelial tumor; TM, thymoma; TC, thymic carcinoma; PD-L1, programmed death- ligand 1; TPS-V, tumor proportion score of PD-L1 using visual evaluation; TPS-IA, tumor proportion score of PD-L1 using image analysis.

\section{Methods}

We collected relevant studies in the last five years (from January 2016 to April 2021) from PubMed by searching for PD-L1 expression and TET, and 21 studies met the requirements. After eliminating the reviews, metaanalyses, and case reports, a total of 14 articles were finally selected. When searching for immune checkpoint inhibitors (ICIs) and TETs, five studies were deemed acceptable. The relevant data were summarized and analyzed. For each study, an electronic literature search was conducted independently and repeatedly by two authors to screen qualified records, review complete articles that might be relevant, and evaluate for inclusion. A list of references for all included studies was manually searched, along with potentially relevant articles. We mainly analyzed the expression levels of PD-L1, median progression-free survival (mPFS) and median overall survival (mOS) values, and irAEs among these studies.

\section{Discussion}

\section{PD1/PD-L1 and CTLA-4 in TETS}

The expression and prognostic value of PD-1/PD-L1 in TETs

Some studies have confirmed that PD-L1 is expressed in TETs. A meta-analysis concluded that PD-L1 was differentially expressed in A/AB/B1 thymoma, B2/B3 thymoma, and thymic carcinoma, and had predictive value for anti-PD-L1 treatment of TETs (28). According to the 14 previous studies on the expression of PD-L1 in TETs in the past five years (Table 1), we can see that all TETs showed expression of PD-L1 (29-42), while TCs could reach 100\% expression in the studies conducted by Bedekovics et al. (30) and Owen et al. (38). In addition, Chen et al. analyzed the expression level of PD-L1 in different types of TETs using The Cancer Genome Atlas database and found that the expression of PD-L1 was higher in TCs than in TMs. In 
Table 2 Main trials results about ICIs in TET patients

\begin{tabular}{|c|c|c|c|c|c|c|c|}
\hline Study & Years & $\begin{array}{l}\text { Experimental } \\
\text { drug }\end{array}$ & $\begin{array}{c}\text { Number of patients } \\
(\mathrm{TM} / \mathrm{TC})\end{array}$ & $\begin{array}{c}\text { mPFS } \\
\text { (months) }\end{array}$ & $\begin{array}{c}\text { mOS (months) } \\
\text { (TM/TC) }\end{array}$ & ORR (TM/TC) & DCR \\
\hline Naziye Ak, et al. (46) & 2020 & Nivolumab & 46 (4/3/1 mixt) & 6.5 & 7.4 & $66.7 \%$ & $100 \%$ \\
\hline Yuki Katsuya, et al. (48) & 2019 & Nivolumab & 15 & 3.8 & 14.1 & $0 \%$ & $73.3 \%$ \\
\hline
\end{tabular}

$\mathrm{ICI}$, immune checkpoint inhibitor; TET, thymic epithelial tumor; TM, thymoma; TC, thymic carcinoma; mPFS, median progression-free suivival; mOS, median overall survival; ORR, overall response rate; DCR, disease control rate.

conclusion, the expression of PD-L1 in TET cells correlates with the degree of tumor malignancy (36). All in all, it can be seen that PD-L1 is highly expressed in TETs, which provides a theoretical basis for the clinical immunotherapy of TETs.

\section{The expression and prognostic value of CTLA-4 in TETs}

Santoni et al. evaluated the correlation between CTLA4 expression and TETs, especially in TMs (43). They evaluated the expression of CTLA-4 in paraffin-embedded tissues of $68 \mathrm{TM}$ patients at mRNA and protein levels. In addition, they evaluated the relevance between the expression of CTLA-4, clinicopathological features, and prognosis in patients with TM. They concluded that in TM patients, CTLA-4 overexpression was significantly associated with reduced OS, thus CTLA-4 was a negative prognostic factor.

\section{The efficacy of immunotherapy in TETs}

Immunotherapy has been applied in many cancer species, and numerous studies have confirmed that its efficacy is positively correlated with the expression of PD-L1. Due to the high expression of PD-L1 in TETs, immunotherapy is expected to achieve a breakthrough efficacy. Yang et al. (44) first reported immunotherapy in TC, and Zander et al. (45) first reported immunotherapy in TM. Subsequently, in the last five years, five available clinical studies have observed the efficacy of immunotherapy in patients with TETs (46-50) (Table 2). Currently, there are no online data about the application of CTLA-4 antibodies in TETs.

Giaccone et al. (50) conducted a phase II clinical study with the largest dataset available to date. Among the 40 patients treated with pembrolizumab, one patient (3\%) achieved complete response (CR), and eight patients (20\%) achieved partial response (PR), with a total overall response rate (ORR) of $22.5 \%$. Furthermore, 21 out of 40 patients $(53 \%)$ had stable disease (SD), with a disease control rate (DCR) of $75 \%$. By the end of the study, 31 patients (78\%) had progressive disease (PD), and 17 patients (43\%) died. The mPFS was 2.4 months and the mOS was 24.9 months. Another study conducted by Cho et al. (49) evaluated pembrolizumab in 26 patients with TC and seven patients with TM. The results showed that the ORR was $28.6 \%$ in the TM group and $19.2 \%$ in the TC group. The DCR was $100 \%$ in the TM group and $73.1 \%$ in the TC group. The mPFS was 6.1 months in both groups. The mOS was 14.5 months in the TC group, but the data did not reach the end point in the TM group.

As for the use of nivolumab in TET patients, Katsuya et al. (48) conducted a single-arm, multicenter, phase II trial to evaluate the efficiency and safety for unresectable or recurrent TC. Among the 15 enrolled patients, 11 had $\mathrm{SD}$, and 4 had PD; therefore, the ORR was $0 \%$, while the DCR was $73.3 \%$. The mPFS was 3.8 months and the mOS was 14.1 months, respectively. The latest study conducted by Ak et al. (46) also treated four TMs, three TCs, and one mixed histology with nivolumab. Two patients' evaluation of best response was not applicable. Among the five available patients, the ORR was $66.7 \%$, and the DCR was $100 \%$. The median follow-up time was 16.1 months. The mPFS and mOS were 6.5 months and 7.4 months, respectively.

In 2019, Rajan et al. (47) conducted a phase I, doseescalation trial of avelumab (MSB0010718C) in seven TM patients and one TC patient. Differently from pembrolizumab and nivolumab, avelumab is a PD-L1 antibody. Two patients got CR, and three patients got SD; the ORR was $25 \%$, and the DCR was $62.5 \%$. PFS and OS were not evaluated in this study.

The above five clinical trials were conducted to determine 


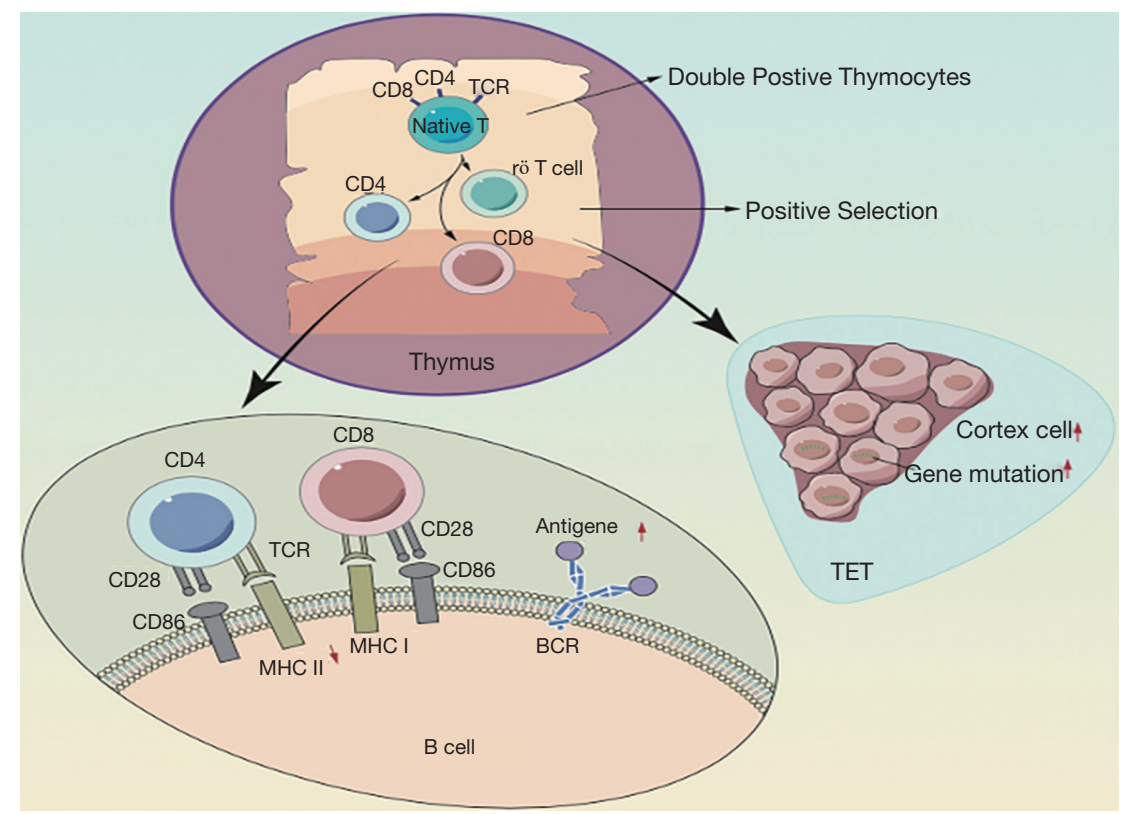

Figure 3 TET patients showed a decline of MHC II, they affected the efficiency of positive selection, then leading T cells with CD4+/ CD8+ double expression to produce too much, so B cells produce antibodies. In addition, higher proliferation in TET cells increased the risk of genetic mutations. TET, thymic epithelial tumor.

whether the PD-1 inhibitors pembrolizumab and nivolumab and the PD-L1 inhibitor avelumab can benefit patients with TETs. They all showed a high ORR and achieved a more significant long-term effect than previous treatment options.

\section{The immune function and autoimmune diseases in TETs}

Several previous studies found that $30 \%$ of patients with TETs have autoimmune diseases, which can occur simultaneously or after surgical removal $(51,52)$. The immune function of the thymus includes two main aspects. First, T cells complete the whole differentiation and maturation processes in the thymus. Second, the thymus is the main organ of the immune system to maintain the stability of its own internal environment and immune tolerance. Based on the understanding of thymic immune function, the researchers concluded that the susceptibility of TET patients to autoimmune diseases was mainly related to immune intolerance. There are two theories regarding the mechanism by which patients become susceptible to autoimmune diseases (Figure 3).

\section{Mechanism of abnormal immune regulation}

TET patients showed a decline in major histocompatibility complex class II molecules, which affected the efficiency of positive selection, leading to excessive production of $\mathrm{T}$ cells with $\mathrm{CD} 4+/ \mathrm{CD} 8+$ double expression so that $\mathrm{B}$ cells produce antibodies and induce disease (53).

\section{Mechanism of susceptibility genes}

In TETs, a large number of cortical thymic cells proliferate, and their increased proliferation increases the risk of genetic mutations since these highly proliferative cortical thymic cells are self-reactive (54).

\section{The safety of immunotherapy in TETs}

As an important immune organ, the thymus gland is the central component of $\mathrm{T}$ cell maturation. TET cells may cause abnormal regulation of $\mathrm{T}$ cells, leading to autoimmune diseases, such as myasthenia gravis, connective tissue diseases, or blood diseases (55). An analysis of 30 years of data from the English literature by Detterbeck and Zeeshan showed that approximately $40 \%$ of thymoma patients were likely to have myasthenia gravis, pure red cell aplasia (PRCA), or parathyroid adenoma (PTA) (56). Bernard et al. retrospectively analyzed clinical data from three French hospitals from 2005 to 2011, where 85 patients 
Table 3 Immune-related adverse events in TET patients

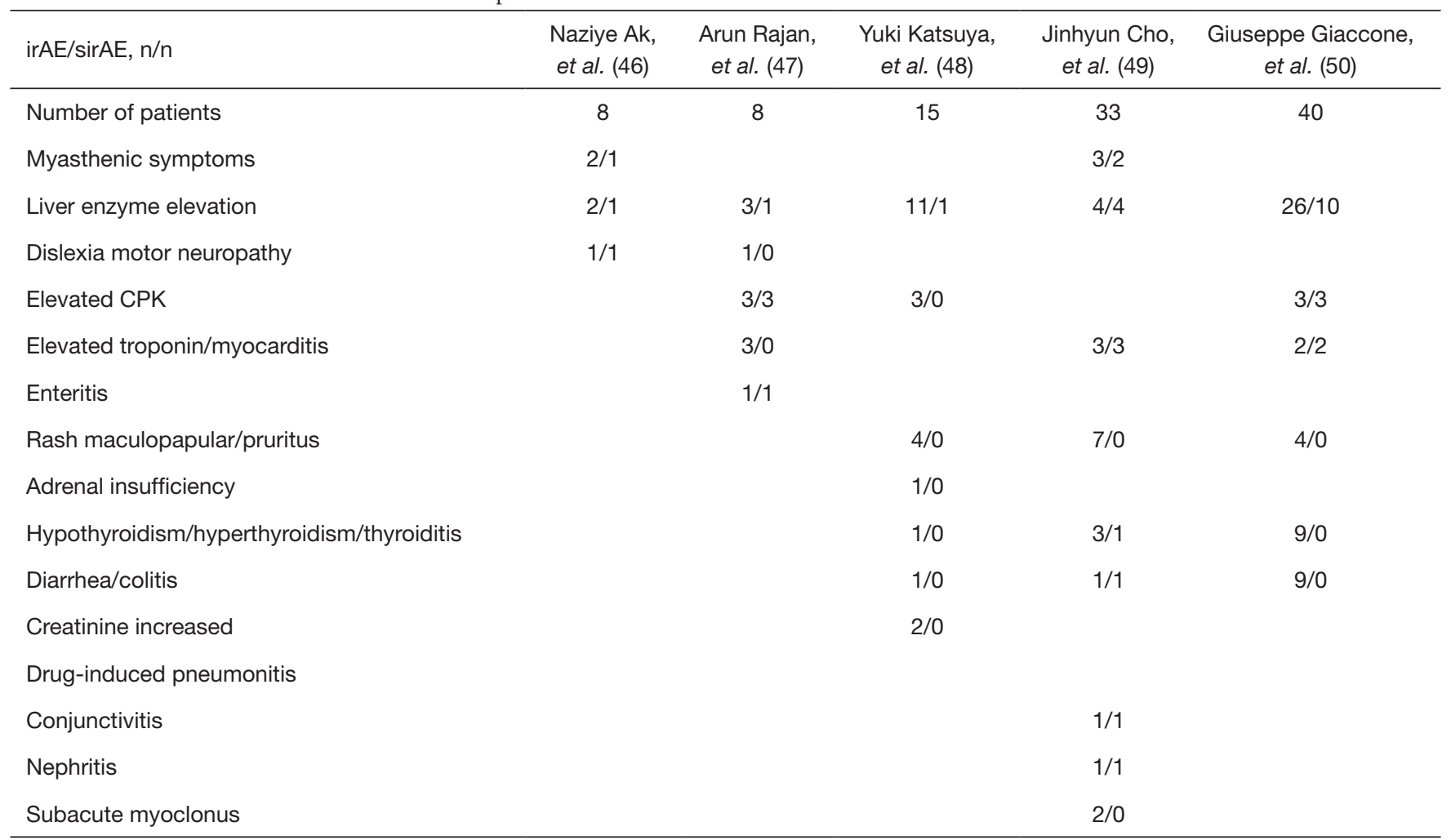

TET, thymic epithelial tumor; CPK, creatine phosphokinase.

with thymoma were enrolled. The results showed that $55 \%$ of patients (47 cases) with TETs had autoimmune diseases developed at the same time, including 33 cases of myasthenia gravis, four cases of Hashimoto thyroiditis, three cases of Isaacs syndrome, two cases of Morvan syndrome, two cases of PRCA, two cases of systemic lupus erythematosus, two cases of lichen planus, and one case of the coexistence of a variety of autoimmune diseases (57). These results suggest that the coexistence of autoimmune diseases such as myasthenia gravis and thymoma is not spontaneous. In addition, a retrospective study conducted by Gutzmer et al. showed that $42 \%$ (8/19) of melanoma patients with a history of autoimmune disease had a recurrence of the original autoimmune disease after using PD-1 inhibitor (58). Therefore, a history of autoimmune diseases may be a susceptibility factor for subsequent autoimmune diseases. The thymus is an immune organ of the human body, so the possibility of myasthenia gravis being caused by the disturbed autoimmune balance in TET patients cannot be ruled out. This may also be the result of the combined effects of the anti-PD-L1 antibody and TETs.
In the last five years, five clinical trials have reported irAEs after immunotherapy in patients with TET (Table 3). As a result of our analysis, we concluded that the most common irAEs were myasthenic symptoms, liver enzyme elevation, elevated creatine phosphokinase, elevated troponin/myocarditis, rash maculopapular/pruritus, hypothyroidism/hyperthyroidism/thyroiditis, and diarrhea/ colitis. The study conducted by Ak et al. (46) enrolled eight patients, two of whom had grade 4 adverse events (AEs), including dyslexia and motor neuropathy, myasthenic symptoms, and liver enzyme elevation, which may have been caused by immunotherapy. As for management, they stopped the administration of an ICI. The study conducted by Rajan $e t a l$. (47) revealed that in a total of eight patients, there were three grade 3 AEs (autoimmune disorder) and three grade 4 AEs (two patients had an autoimmune disorder and one had hypokalemia). All patients received steroids, and they were not re-treated with avelumab. Katsuya et al. (48) reported a study that included 15 patients, two of whom experienced immune-related serious AEs; one patient had grade III aspartate aminotransferase 
increase, and the other had grade II adrenal insufficiency. The former patient discontinued nivolumab treatment and recovered after 19 days. The other patients were treated with hydrocortisone. Cho et al. (49) conducted an openlabel phase II trial that included 33 patients. Five out of seven patients with TM had grade 3-4 irAEs, including two hepatitis, three myocarditis, one thyroiditis, one colitis, one conjunctivitis, and one nephritis. Four out of 26 patients with TC had grade 3-4 irAEs, including two hepatitis, two myasthenia gravis, and one subacute myoclonus. Most of these patients discontinued treatment with pembrolizumab and recovered with the treatment of highdose corticosteroids and other immunosuppressive agents. Giaccone et al. (50) also conducted a phase II study that enrolled 41 patients; six patients had one or more severe irAEs. Grade 3 or 4 AEs included five cases of increased aspartate aminotransferase, five of alanine aminotransferase, three of dyspnea, three of myalgia or myositis, three of increased creatine phosphokinase, two of anemia, two of myocarditis, one of arthralgia, one of blurred vision, one of hyperglycemia, one of increased lipase, and one case of thrombocytopenia. Four of these six patients were treated with intravenous steroids followed by high-dose oral steroids.

According to the above data, for the management of mild irAEs following the use of ICIs, such as grade I rash and grade I transaminase elevations, symptomatic treatment is sufficient, and discontinuing the treatment is not necessary. As for the management of severe irAEs, discontinuation of ICIs and treatment with immunosuppressant glucocorticoids are required. Other immunosuppressive agents, such as tacrolimus and cyclosporine, may be considered if symptoms do not improve significantly or continue to worsen. Due to the relatively high incidence of irAEs, especially in TM patients, the use of ICIs should be avoided as much as possible. Early detection and management of irAEs are also critical for patients with immunotherapy compliance and TC.

\section{Conclusions}

With the in-depth research and development of immunotherapy, immunotherapy drugs such as PD-1 and PD-L1 inhibitors were found to be effective in TET patients. The current National Comprehensive Cancer Network guidelines have recommended pembrolizumab as a second-line treatment for TC, with the level of evidence of class 2A. Therefore, immunotherapy could be used in the treatment of TETs, especially in the absence of standard second-line treatment for TCs. The use of immunotherapy provides patients with new opportunities and hope. However, due to the specificity of TETs and immunity, enough attention should also be paid to potential irAEs caused by immunotherapy.

As for the exploration of the efficacy and safety of immunotherapy, the results showed that the expression level of PD-L1 might be positively correlated with the response rate of immunotherapy. However, further confirmation with larger sample sizes is needed, and other biomarkers should be explored. A considerable proportion of patients with TETs are complicated with autoimmune syndromes, such as myasthenia gravis and Hashimoto's thyroiditis. Although there were many patients with high PD-L1 expression in TETs, and the immunotherapy seemed promising, we still need to pay more attention to the occurrence of irAEs. The benefits and risks of immunotherapy for patients with TETs still need to be measured in further studies. Due to the limited data, we will still pay attention to future articles on TETs and continue to improve our implications.

\section{Acknowledgments}

Funding: This work was supported by the National Natural Science Foundation of China (81802255), Young Talents in Shanghai (2019QNBJ), 'Dream Tutor' Outstanding Young Talents Program (fkyq1901), Clinical Research Project of Shanghai Pulmonary Hospital (fk18005), Key Discipline in 2019 (oncology), Project of Shanghai Municipal Science and Technology Commission (Project of Municipal Science and Technology Commission), Scientific research project of Shanghai Pulmonary Hospital (fkcx1903), Shanghai Municipal Commission of Health and Family Planning (2017YQ050), Innovation Training Project of SITP of Tongji University, and key projects of leading talent (19411950300). Youth project of hospital management research fund of Shanghai Hospital Association (Q1902037).

\section{Footnote}

Reporting Checklist: The authors have completed the PRISMA reporting checklist. Available at https://dx.doi. org/10.21037/jtd-21-290

Conflicts of Interest: All authors have completed the ICMJE uniform disclosure form (available at https://dx.doi. org/10.21037/jtd-21-290). YH receives funding for the payment for publication from National Natural Science Foundation of China (81802255) and Clinical research 
project of Shanghai Pulmonary Hospital (FKLY20010). The other authors have no conflicts of interest to declare.

Ethical Statement: The authors are accountable for all aspects of the work in ensuring that questions related to the accuracy or integrity of any part of the work are appropriately investigated and resolved.

Open Access Statement: This is an Open Access article distributed in accordance with the Creative Commons Attribution-NonCommercial-NoDerivs 4.0 International License (CC BY-NC-ND 4.0), which permits the noncommercial replication and distribution of the article with the strict proviso that no changes or edits are made and the original work is properly cited (including links to both the formal publication through the relevant DOI and the license). See: https://creativecommons.org/licenses/by-nc-nd/4.0/.

\section{References}

1. Engels EA, Pfeiffer RM. Malignant thymoma in the United States: demographic patterns in incidence and associations with subsequent malignancies. Int J Cancer 2003;105:546-51.

2. Yokoi K, Kondo K, Fujimoto K, et al. JLCS medical practice guidelines for thymic tumors: summary of recommendations. Jpn J Clin Oncol 2017;47:1119-22.

3. Wright CD, Wain JC, Wong DR, et al. Predictors of recurrence in thymic tumors: importance of invasion, World Health Organization histology, and size. J Thorac Cardiovasc Surg 2005;130:1413-21.

4. Yang L, Cai W, Yang X, et al. Development of a deep learning model for classifying thymoma as Masaoka-Koga stage I or II via preoperative CT images. Ann Transl Med 2020;8:287.

5. Margaritora S, Cesario A, Cusumano G, et al. Thirty-fiveyear follow-up analysis of clinical and pathologic outcomes of thymoma surgery. Ann Thorac Surg 2010;89:245-52; discussion 252.

6. Okuma Y, Hosomi Y, Takagi Y, et al. Clinical outcomes with chemotherapy for advanced thymic carcinoma. Lung Cancer 2013;80:75-80.

7. Lemma GL, Lee JW, Aisner SC, et al. Phase II study of carboplatin and paclitaxel in advanced thymoma and thymic carcinoma. J Clin Oncol 2011;29:2060-5.

8. Hirai F, Yamanaka T, Taguchi K, et al. A multicenter phase II study of carboplatin and paclitaxel for advanced thymic carcinoma: WJOG4207L. Ann Oncol 2015;26:363-8.
9. Bluthgen MV, Boutros C, Fayard F, et al. Activity and safety of oral etoposide in pretreated patients with metastatic or recurrent thymic epithelial tumors (TET): A single-institution experience. Lung Cancer 2016;99:111-6.

10. Giaccone G, Ardizzoni A, Kirkpatrick A, et al. Cisplatin and etoposide combination chemotherapy for locally advanced or metastatic thymoma. A phase II study of the European Organization for Research and Treatment of Cancer Lung Cancer Cooperative Group. J Clin Oncol 1996;14:814-20.

11. Thomas A, Rajan A, Berman A, et al. Sunitinib in patients with chemotherapy-refractory thymoma and thymic carcinoma: an open-label phase 2 trial. Lancet Oncol 2015;16:177-86.

12. Zucali PA, De Pas T, Palmieri G, et al. Phase II Study of Everolimus in Patients With Thymoma and Thymic Carcinoma Previously Treated With Cisplatin-Based Chemotherapy. J Clin Oncol 2018;36:342-9.

13. Xia L, Huang H, Xiao H, et al. Utilization of combined PD-L1 expression and neutrophil-to-lymphocyte ratio prior to surgery as a prognostic factor in non-small cell lung cancer with brain metastasis. Transl Cancer Res 2019;8:2864-77.

14. Reck M, Rodríguez-Abreu D, Robinson AG, et al. Pembrolizumab versus Chemotherapy for PD-L1Positive Non-Small-Cell Lung Cancer. N Engl J Med 2016;375:1823-33.

15. Xu Y, Wan B, Chen X, et al. The association of PDL1 expression with the efficacy of anti-PD-1/PD-L1 immunotherapy and survival of non-small cell lung cancer patients: a meta-analysis of randomized controlled trials. Transl Lung Cancer Res 2019;8:413-28.

16. Seiwert TY, Burtness B, Mehra R, et al. Safety and clinical activity of pembrolizumab for treatment of recurrent or metastatic squamous cell carcinoma of the head and neck (KEYNOTE-012): an open-label, multicentre, phase $1 \mathrm{~b}$ trial. Lancet Oncol 2016;17:956-65.

17. Chen G, Huang AC, Zhang W, et al. Exosomal PD-L1 contributes to immunosuppression and is associated with anti-PD-1 response. Nature 2018;560:382-6.

18. Yokosuka T, Takamatsu M, Kobayashi-Imanishi W, et al. Programmed cell death 1 forms negative costimulatory microclusters that directly inhibit $\mathrm{T}$ cell receptor signaling by recruiting phosphatase SHP2. J Exp Med 2012;209:1201-17.

19. Sharpe AH, Pauken KE. The diverse functions of the PD1 inhibitory pathway. Nat Rev Immunol 2018;18:153-67.

20. Salama AK, Hodi FS. Cytotoxic T-lymphocyte-associated 
antigen-4. Clin Cancer Res 2011;17:4622-8.

21. Chuang E, Fisher TS, Morgan RW, et al. The CD28 and CTLA-4 receptors associate with the serine/threonine phosphatase PP2A. Immunity 2000;13:313-22.

22. Stein PH, Fraser JD, Weiss A. The cytoplasmic domain of CD28 is both necessary and sufficient for costimulation of interleukin-2 secretion and association with phosphatidylinositol 3'-kinase. Mol Cell Biol 1994;14:3392-402.

23. Linsley PS, Greene JL, Tan P, et al. Coexpression and functional cooperation of CTLA-4 and CD28 on activated T lymphocytes. J Exp Med 1992;176:1595-604.

24. Chuang E, Alegre ML, Duckett CS, et al. Interaction of CTLA-4 with the clathrin-associated protein AP50 results in ligand-independent endocytosis that limits cell surface expression. J Immunol 1997;159:144-51.

25. van der Merwe PA, Bodian DL, Daenke S, et al. CD80 (B7-1) binds both CD28 and CTLA-4 with a low affinity and very fast kinetics. J Exp Med 1997;185:393-403.

26. Martin M, Schneider H, Azouz A, et al. Cytotoxic T lymphocyte antigen 4 and CD28 modulate cell surface raft expression in their regulation of $T$ cell function. J Exp Med 2001;194:1675-81.

27. Schneider H, Downey J, Smith A, et al. Reversal of the TCR stop signal by CTLA-4. Science 2006;313:1972-5.

28. Chen HF, Wu LX, Li XF, et al. PD-L1 expression level in different thymoma stages and thymic carcinoma: a metaanalysis. Tumori 2020;106:306-11.

29. Berardi R, Goteri G, Brunelli A, et al. Prognostic relevance of programmed cell death protein 1/programmed deathligand 1 pathway in thymic malignancies with combined immunohistochemical and biomolecular approach. Expert Opin Ther Targets 2020;24:937-43.

30. Bedekovics J, Beke L, Mokanszki A, et al. Programmed Death-ligand 1 (PD-L1) Expression in Thymic Epithelial Tumors. Appl Immunohistochem Mol Morphol 2020;28:1-9.

31. Ishihara S, Okada S, Ogi H, et al. Programmed deathligand 1 expression profiling in thymic epithelial cell tumors: Clinicopathological features and quantitative digital image analyses. Lung Cancer 2020;145:40-7.

32. Rouquette I, Taranchon-Clermont E, Gilhodes J, et al. Immune biomarkers in thymic epithelial tumors: expression patterns, prognostic value and comparison of diagnostic tests for PD-L1. Biomark Res 2019;7:28.

33. Higuchi R, Goto T, Hirotsu Y, et al. PD-L1 Expression and Tumor-Infiltrating Lymphocytes in Thymic Epithelial Neoplasms. J Clin Med 2019;8:1833.
34. Song JS, Kim D, Kwon JH, et al. Clinicopathologic Significance and Immunogenomic Analysis of Programmed Death-Ligand 1 (PD-L1) and Programmed Death 1 (PD1) Expression in Thymic Epithelial Tumors. Front Oncol 2019;9:1055.

35. Funaki S, Shintani Y, Fukui E, et al. The prognostic impact of programmed cell death 1 and its ligand and the correlation with epithelial-mesenchymal transition in thymic carcinoma. Cancer Med 2019;8:216-26.

36. Chen Y, Zhang Y, Chai X, et al. Correlation between the Expression of PD-L1 and Clinicopathological Features in Patients with Thymic Epithelial Tumors. Biomed Res Int 2018;2018:5830547.

37. Duan J, Liu X, Chen H, et al. Impact of PD-L1, transforming growth factor- $\beta$ expression and tumorinfiltrating CD8+ T cells on clinical outcome of patients with advanced thymic epithelial tumors. Thorac Cancer 2018;9:1341-53.

38. Owen D, Chu B, Lehman AM, et al. Expression Patterns, Prognostic Value, and Intratumoral Heterogeneity of PDL1 and PD-1 in Thymoma and Thymic Carcinoma. J Thorac Oncol 2018;13:1204-12.

39. Arbour KC, Naidoo J, Steele KE, et al. Expression of PD-L1 and other immunotherapeutic targets in thymic epithelial tumors. PLoS One 2017;12:e0182665.

40. Weissferdt A, Fujimoto J, Kalhor N, et al. Expression of PD-1 and PD-L1 in thymic epithelial neoplasms. Mod Pathol 2017;30:826-33.

41. Marchevsky AM, Walts AE. PD-L1, PD-1, CD4, and CD8 expression in neoplastic and nonneoplastic thymus. Hum Pathol 2017;60:16-23.

42. Katsuya Y, Horinouchi H, Asao T, et al. Expression of programmed death 1 (PD-1) and its ligand (PD-L1) in thymic epithelial tumors: Impact on treatment efficacy and alteration in expression after chemotherapy. Lung Cancer 2016;99:4-10.

43. Santoni G, Amantini C, Morelli MB, et al. High CTLA4 expression correlates with poor prognosis in thymoma patients. Oncotarget 2018;9:16665-77.

44. Yang Y, Ding L, Wang P. Dramatic response to antiPD-1 therapy in a patient of squamous cell carcinoma of thymus with multiple lung metastases. J Thorac Dis 2016;8:E535-7.

45. Zander T, Aebi S, Rast AC, et al. Response to Pembrolizumab in a Patient with Relapsing Thymoma. J Thorac Oncol 2016;11:e147-9.

46. Ak N, Aydiner A. Nivolumab treatment for metastatic thymic epithelial tumors. J Oncol Pharm Pract 2020. [Epub 
ahead of print]. doi: 10.1177/1078155220968150.

47. Rajan A, Heery CR, Thomas A, et al. Efficacy and tolerability of anti-programmed death-ligand 1 (PD-L1) antibody (Avelumab) treatment in advanced thymoma. J Immunother Cancer 2019;7:269.

48. Katsuya Y, Horinouchi H, Seto T, et al. Single-arm, multicentre, phase II trial of nivolumab for unresectable or recurrent thymic carcinoma: PRIMER study. Eur J Cancer 2019;113:78-86.

49. Cho J, Kim HS, Ku BM, et al. Pembrolizumab for Patients With Refractory or Relapsed Thymic Epithelial Tumor: An Open-Label Phase II Trial. J Clin Oncol 2019;37:2162-70.

50. Giaccone G, Kim C, Thompson J, et al. Pembrolizumab in patients with thymic carcinoma: a single-arm, singlecentre, phase 2 study. Lancet Oncol 2018;19:347-55.

51. Jamilloux Y, Frih H, Bernard C, et al. Thymoma and autoimmune diseases. Rev Med Interne 2018;39:17-26.

52. Fujii Y. Thymus, thymoma and myasthenia gravis. Surg Today 2013;43:461-6.

Cite this article as: Song X, Fan J, Zhu L, Wang Z, He Y, Zhou C. The efficacy and safety of immunotherapy in thymic epithelial tumors: more effective, more risky: a systematic review. J Thorac Dis 2021;13(8):5093-5103. doi: 10.21037/ jtd-21-290
53. Evoli A, Minicuci GM, Vitaliani R, et al. Paraneoplastic diseases associated with thymoma. J Neurol 2007;254:756-62.

54. Okumura M, Fujii Y, Shiono H, et al. Immunological function of thymoma and pathogenesis of paraneoplastic myasthenia gravis. Gen Thorac Cardiovasc Surg 2008;56:143-50.

55. Scheffzek K, Ahmadian MR, Kabsch W, et al. The Ras-RasGAP complex: structural basis for GTPase activation and its loss in oncogenic Ras mutants. Science 1997;277:333-8.

56. Detterbeck FC, Zeeshan A. Thymoma: current diagnosis and treatment. Chin Med J (Engl) 2013;126:2186-91.

57. Bernard C, Frih H, Pasquet F, et al. Thymoma associated with autoimmune diseases: 85 cases and literature review. Autoimmun Rev 2016;15:82-92.

58. National Comprehensive Cancer Network. Malignant pleural mesothelioma guidelines. Available online: https:// www.nccn.org/professionals/physician_gls/pdf/mpm_ blocks.pdf. Accessed March 19, 2019. 